\title{
Conocimientos, actitudes y prácticas sobre dengue en estudiantes de educación primaria en Chorrillos, Lima, Perú
}

Knowledge, attitudes and practices about dengue fever in elementary school students in Chorrillos, Lima, Peru

\author{
Rufino Cabrera ${ }^{1}$, Andrea Gómez de la Torre-Del Carpio ${ }^{1}$, Alejandra Isabel \\ Bocanegra Jesús ${ }^{1}$, Jorge Mauricio Correa Borit ${ }^{1}$, Francisco Javier Huamaní Fuente ${ }^{1}$, \\ Paula Valeria Urrunaga Poma ${ }^{1}$, Elsa Ibarra-Casablanca ${ }^{1}$ \\ 'Escuela de Medicina, Universidad Peruana de Ciencias Aplicadas, Lima, Perú.
}

\begin{abstract}
Resumen
Objetivos. Evaluar los conocimientos, actitudes y prácticas sobre dengue, medidas de prevención y control, e identificar los factores asociados a su desconocimiento, en alumnos de una institución educativa estatal. Diseño. Estudio observacional transversal. Lugar. Institución educativa estatal en Chorrillos, Lima, Perú. Participantes. Alumnos de educación primaria. Métodos. En el año 2013, se encuestaron 142 alumnos del quinto y sexto grado de educación primaria y se exploró la percepción en 12 brigadieres en base a la técnica proyectiva. Principales medidas de resultados. Conocimientos, actitudes y prácticas sobre dengue. Resultados. El 53,5\% (76) era varón y el promedio de edad fue 11,2 con $\pm 1,05$ años. El 33,1 \% (47; IC $95 \% 25,4$ a 41,4) de los estudiantes tenía un conocimiento básico de dengue y $53,5 \%$ (76) identificaron la forma de transmisión vectorial del dengue. El 29,6 \% (42; IC95\% 22,2 a 37,8) de los escolares reconocieron las larvas y $54,2 \%$ (77) la forma adulta del vector. El $5,6 \%$ (8) y 2,1\% (3) de los encuestados identificaron el larvicida y el cambio de arena húmeda de los floreros como medidas de prevención de la infestación domiciliaria. El 75,4\% (75; IC95\% 67,4 a 82,2) de los niños aceptarían participar en la búsqueda del vector, con diferencias significativas entre los niños y las niñas $(p=0,049238)$. El no haber recibido información previa se asoció a no tener conocimiento básico de dengue $(\mathrm{OR}=3,7 ; \mathrm{IC} 95 \%$ $1,7$ a 8,$3 ; p=0,0004676)$. Conclusiones. Los conocimientos de los estudiantes sobre los signos y sintomas y la forma de transmisión del dengue, las características del vector y las medidas de prevención del dengue fueron bajos y el no recibir información se asoció al desconocimiento del dengue.
\end{abstract}

Palabras clave. Dengue, Conocimientos, Actitudes y Práctica en Salud; Estudiantes; Técnicas Proyectivas.

\section{Abstract}

Objectives. To assess the knowledge, attitudes and practices on dengue fever, measures for prevention and control, and to identify factors related to unawareness in public elementary school students in the district of Chorrillos, Lima, Peru. Design. Cross sectional observational study. Setting. State elementary institution in Chorrillos district, Lima, Peru. Participants. Students of elementary school. Methods. In the year 2013, 142 students from fifth and sixth grade answered a survey questionnaire. We explored the perception among 12 school brigadiers using the projective technique. Main outcome measures. Knowledge, attitudes and practices about dengue. Results. In the study, 53.5\% students were boys (76), mean age $11.2 \pm 1.05$ years old; $33.1 \%$ (47; Cl95\% 25.4-41.4) had basic knowledge about dengue; $53.5 \%$ (76) were able to identify dengue virus' mechanism of vertical transmission; $29.6 \%$ of students (42; Cl95\% 22.2-37.8) were able to identify the larvae and $54.2 \%$ (77) identified the adult flying mosquito. Only $5.6 \%$ (8) and 2.1\% (3) knew respectively of larvicides and using wet sand in flower vases as measures of control at home; $75.4 \%$ of the boys (75; Cl95\% 67.4-82.2) were willing to participate in active control of the mosquito vector, with statistical difference between boys and girls $(p=0.049238)$. Not having previous information led to not having basic knowledge about dengue ( $O R=3.7$; Cl95\% 1.7-8.3) $(p=0.0004676)$ Conclusion. The knowledge among students about clinical features, mode of transmission, vector and measures of prevention and control of the dengue virus were low because they had not received appropriate information.

Keywords. Dengue, Knowledge, Attitudes, Practice; Students; Projective Techniques.

An Fac med. 2016;77(2):129-35 / http://dx.doi.org/10.15381/anales.v77i2.11817

\section{INTRODUCCIÓN}

El dengue es una de las enfermedades virales re-emergentes trasmitidas por mosquitos más importantes en el mundo. Se estima entre 50 y 200 millones de infectados al año, de los cuales, 500000 desarrollan dengue grave, y se reportan más de 20000 defunciones por año ${ }^{(1)}$.
En América Latina, la mayoría de los países son endémicos o hiperendémicos; solo Uruguay y Chile continental no informan sobre casos autóctonos ${ }^{(1)}$. Entre 2004 y 2010, los países con mayor número de casos reportados en el mundo fueron Brasil, Indonesia y Vietnam; Perú ocupó el puesto $18^{(2)}$.

En 1984, se reintrodujo el Aedes aegypti al Perú y seis años más tarde se presentaron los primeros grandes brotes de dengue asociados al serotipo DENV. 1 y DENV-4, en las ciudades de Iquitos, Tarapoto y Moyobamba, ubicadas en la región nororiental del Perú ${ }^{(3)}$. Desde entonces, el dengue se ha expandido a otras regiones del país. En el 2000, se informó sobre la reinfestación por Ae. aegypti de la ciudad de Lima, en el distrito del Rímac ${ }^{(4)}$, y en el 2005 ocurrió 
el primer brote de dengue en Lima, en el distrito de Comas ${ }^{(5)}$.

Antes de 2013, la mayoría de los brotes de dengue en la ciudad de Lima se presentaban, principalmente, en los distritos de Puente Piedra ${ }^{(6)}$, Independencia, Comas y en San Juan de Lurigancho; sin embargo, en el 2013, se presentó por primera vez un brote en el distrito de Villa María del Triunfo, jurisdicción de la Dirección de Salud Lima Sur (DISA Lima Sur), donde 7 de 13 distritos se encuentran infestados con el vector. En enero de 2013, se comunicó la infestación en el distrito de Chorrillos ${ }^{(7)}$.

Por otro lado, los principales determinantes para la presentación de brotes de dengue en la ciudad de Lima, entre otros, serían la subestimación de los índices aédicos, la baja cobertura de agua dentro de las viviendas que obliga a almacenar agua en los depósitos ${ }^{(7)}$, el escaso conocimiento de las medidas de prevención y control de la enfermedad por la población ${ }^{(8)}$, las bajas coberturas de tratamiento focal, entre otros.

Se han realizado varios estudios en otros países endémicos de la región ${ }^{(9-12)}$ así como en el Perú ${ }^{(8,13,14)}$, sobre la percepción de la población adulta sobre los signos y síntomas del dengue, el vector, su hábitat y ciclo biológico, la participación comunitaria y las medidas de prevención y el control sobre la enfermedad. Sin embargo, existen escasos estudios que han evaluado la percepción de escolares sobre el dengue y las medidas de prevención ${ }^{(15-20)}$, a pesar que esta población tiene un rol importante en la vigilancia entomológica. En el Perú, no existe algún estudio al respecto.

Por ello, el objetivo del estudio fue evaluar los conocimientos, actitudes y prácticas sobre el dengue y las medidas de prevención y control e identificar factores asociados al desconocimiento del dengue en alumnos de una institución educativa estatal en el distrito de Chorrillos, Lima, Perú.

\section{MÉTODOS}

El estudio es observacional descriptivotransversal, realizado entre agosto y noviembre de 2013. Se aplicó una encuesta de conocimientos, actitudes y prácticas (CAP) sobre dengue en alumnos del quinto y sexto grado de educación primaria de una institución educativa estatal en el distrito de Chorrillos. Adicionalmente, se realizó un estudio cualitativo para explorar la percepción sobre el dengue en base a dibujos (técnicas proyectivas).

La institución educativa donde se realizó el estudio se ubica en la localidad de San Genaro, en la colina del Morro Solar en el distrito de Chorrillos, al sur de la ciudad de Lima, Perú. La población estimada para el 2013 de Chorrillos fue 318483 habitantes (21). En 2007, este distrito tenía 10938 viviendas sin agua y/o saneamiento, de las cuales $30,6 \%, 24,4 \%$ y $45 \%$ no contaban con agua, saneamiento básico y ningún servicio, respectivamente ${ }^{(22)}$.

En el 2013, la institución educativa tenía 1674 alumnos matriculados en tres niveles, de ellos 820 en educación primaria (270 en quinto y sexto grado). Para la encuesta CAP, se realizó un muestreo aleatorio simple con asignación proporcional por aula, considerando una prevalencia esperada de $20 \%$ de desconocimiento de los signos y síntomas y la forma de transmisión del dengue, con una precisión de $5 \%$ y un nivel de confianza de $95 \%$. Además, se agregó una tasa de rechazo de $10 \%$, obteniendo una muestra final de 142 alumnos.

Se consideró como criterios de inclusión ser niño o niña, estar matriculados en quinto o sexto grado de educación primaria en el año escolar 2013 y asistir regularmente. Para el estudio cualitativo, se consideraron los criterios previos y ser brigadier de su respectiva aula.

Para el estudio CAP se utilizó una encuesta estructurada anónima de 40 preguntas (abiertas, dicotómicas y de opción múltiple) que contenía datos generales (demográficos y socio-econó- micos) de los participantes y sus jefes de familia; conocimientos sobre los signos y síntomas del dengue, forma de transmisión, características morfológicas y sitios de reproducción del vector; además, actitudes y prácticas sobre uso de medios de información, participación comunitaria, medidas de prevención y control del dengue.

Se definió como conocimiento básico sobre el dengue cuando el alumno reconocía la fiebre como signo principal más otro síntoma como cefalea, mialgia, dolor retro-ocular, artralgias, exantema o vómitos y la forma de transmisión. Para el reconocimiento del vector se utilizó imágenes de formas adultas de diferentes géneros de mosquitos (Anopheles, Aedes aegypti y Culex spp) y muestras conservadas de larvas y pupas de Ae. aegypti.

Con la información obtenida, se elaboró una base de datos en Microsoft Office Excel 2007. El análisis se realizó en MS-Excel y en Epilnfo 7 del Center for Disease and Control, EE UU. Los resultados de las variables cualitativas se mostraron en frecuencias absolutas y relativas y las variables numéricas mediante las medidas de resumen. Para el análisis bivariado, se utilizó el odds ratio (OR), considerando un valor de 'p' menor de 0,05 como significativo y un nivel de confianza al 95\% con la prueba exacta de Fisher.

Para el estudio cualitativo, se seleccionaron 12 brigadieres de quinto y sexto grado de educación primaria, a los cuales se les entregó cartulinas blancas, lápices de colores y borrador y se les pidió que dibujaran la forma de transmisión del dengue, el ciclo biológico del vector y la forma de almacenamiento del agua en sus domicilios. Los dibujos fueron realizados en un ambiente sin la ayuda de los profesores y después de una hora se recogieron y fueron analizados para ver si había consistencia con la encuesta.

El estudio no fue sometido a un comité de ética por tratarse de una encuesta anónima y de bajo riesgo para el participante; sin embargo, el estudio 
fue autorizado por la Institución Educativa, por ser un estudio de interés en la formación de los alumnos. Adicionalmente, se aplicó el asentimiento libre de los alumnos para participar en el estudio. En la encuesta se evitó la identificación de los participantes, excepto en los dibujos.

\section{RESULTADOS}

De los 142 alumnos, 53,5\% (76) eran de sexto y $46,4 \%$ (66) de quinto grado de educación primaria. El 53,5 \% (76) era varón. El promedio de edad fue 11,2 años, rango 8 a 14 , con una $\mathrm{DE} \pm$ 1,05 y una mediana de 11 años.

El 78,1\% (111) de los escolares encuestados nacieron en el departamento de Lima, 3,5\% (5) en Ancash y en el mismo porcentaje en Cusco; 2,8\% (4) en Junín y 12 (11,9\%) en otros departamentos. El jefe de familia en 62\% (88) de los escolares era el papá, en $26,8 \%$ (38) la mamá y en 6,3\% (9) otros familiares cercanos. Respecto a la ocupación laboral del jefe de familia de los escolares, $16,2 \%$ (23) era chofer, $21,1 \%$ (30) comerciante, $14,8 \%$ (21) albañil, $7,7 \%$ (11) ama de casa y 40,1\% (57) tenía otras ocupaciones. Por otro lado, Chorrillos es el distrito donde laboraba el 67,6\% (96) de los jefes de familia de los escolares, en $5,6 \%$ (8) laboraba en San Juan de Miraflores, 2,1\% (3) en Villa María del Triunfo y la misma proporción en Santiago de Surco, 1,4\% (2) en Lurín, Barranco y Lima y el resto, $18,3 \%$ (26), en otros distritos.

El 33,1\% (47; IC95\% 25,4 a 41,4) tenía un conocimiento básico de dengue y no se encontraron diferencias significativas entre las niñas y los niños (valor $\mathrm{p}=0,41579$ ) ni en el grado educativo (valor $\mathrm{p}=0,55905$ ). El 68,3\% (97) consideró que el dengue es una enfermedad grave. La identificación de la sintomatología, la forma de transmisión, reconocimiento de la forma adulta y sitios de reproducción del vector, medidas de prevención y control se muestran en la tabla 1. Respecto a la identificación de las formas larvarias
Tabla 1. Conocimientos sobre los sintomas y signos, formas de transmisión, medidas de prevención y control de dengue y prácticas de riesgo de los escolares de una institución educativa en el distrito de Chorrillos, Lima, Perú.

\begin{tabular}{|c|c|c|}
\hline Variables & $\mathrm{n}(\%)$ & IC $95 \%$ \\
\hline \multicolumn{3}{|l|}{ Signos y síntomas } \\
\hline Fiebre & $93(65,5)$ & 57,1 a 73,3 \\
\hline Fiebre más otro síntoma & $83(58,5)$ & 59,9 a 75,8 \\
\hline Cefalea & $72(50,7)$ & 42,2 a 59,2 \\
\hline Mialgia & $21(14,8)$ & 9,4 a 21,7 \\
\hline Dolor abdominal & $14(9,9)$ & 5,5 a 15,9 \\
\hline Artralgias & $13(9,2)$ & 4,9 a 15,2 \\
\hline Vómitos & $11(7,7)$ & 3,4 a 13,4 \\
\hline Dolor retro-ocular & $7(4,9)$ & 2,0 a 9,8 \\
\hline Exantema & $3(2,1)$ & 0,4 a 6,1 \\
\hline Epistaxis & $1(0,7)$ & 0,02 a 3,8 \\
\hline No reconoce & $105(73,9)$ & 65,9 a 80,9 \\
\hline \multicolumn{3}{|l|}{ Formas de transmisión } \\
\hline Por la picadura de moquitos/zancudos & $76(53,5)$ & 44,9 a 61,9 \\
\hline Tomar agua contaminada & $35(24,6)$ & 17,8 a 32,5 \\
\hline No reconoce & $22(15,6)$ & 9,9 a 22,5 \\
\hline Persona a persona & $8(5,6)$ & 2,4 a 10,8 \\
\hline Por transfusión de sangre & $1(0,7)$ & 0,02 a 3,8 \\
\hline \multicolumn{3}{|l|}{ Reconocimiento de la forma adulta del vector } \\
\hline Vector del dengue (Aedes aegypti) & $77(54,2)$ & 45,6 a 62,6 \\
\hline Culex & $26(18,3)$ & 12,3 a 25,6 \\
\hline Anopheles & $24(16,9)$ & 11,1 a 24,1 \\
\hline No reconoce & $15(10,6)$ & 6,03 a 16,8 \\
\hline \multicolumn{3}{|l|}{ Recipientes donde se reproduce } \\
\hline Tanques de agua & $99(69,7)$ & 61,4 a 77,1 \\
\hline Baldes/Tinas & $59(41,5)$ & 33,3 a 50,1 \\
\hline Cilindros/Sansones/ Bidones & $57(40,1)$ & 32,0 a 48,6 \\
\hline Floreros & $55(38,7)$ & 30,6 a 47,2 \\
\hline Recipientes en desuso & $20(14,1)$ & 8,8 a 20,9 \\
\hline Maceteros & $18(12,7)$ & 7,6 a 19,2 \\
\hline No reconoce & $14(9,9)$ & 5,5 a 15,9 \\
\hline Bebederos & $8(5,63)$ & 2,4 a 10,8 \\
\hline \multicolumn{3}{|l|}{ Métodos para evitar la reproducción del vector } \\
\hline Tapar los recipientes & $105(73,9)$ & 65,9 a 80,9 \\
\hline Escobillar los recipientes & $101(72,1)$ & 62,9 a 78,4 \\
\hline No sabe & $15(10,6)$ & 6,03 a 16,8 \\
\hline Abate ${ }^{\circledR}$ & $8(5,6)$ & 2,4 a 10,8 \\
\hline Eliminar los objetos que almacenan agua & $5(3,4)$ & 1,1 a 8,03 \\
\hline Reemplazar arena húmeda por agua en los floreros & $3(2,1)$ & 0,4 a 6,05 \\
\hline \multicolumn{3}{|l|}{ Métodos para evitar la picadura por mosquitos } \\
\hline Fumigar la vivienda & $58(40,9)$ & 32,6 a 49,4 \\
\hline Usar repelentes o repelente natural & $50(35,2)$ & 27,4 a 43,6 \\
\hline No reconoce & $30(21,1)$ & 14,7 a 28,7 \\
\hline Usar ropa de manga larga & $21(14,8)$ & 9,4 a 21,7 \\
\hline Dormir con mosquiteros & $13(9,1)$ & 4,9 a 15,1 \\
\hline \multicolumn{3}{|l|}{ Abastecimiento de agua en la vivienda de los alumnos } \\
\hline Caño dentro del domicilio por red pública & $95(66,9)$ & 58,5 a 74,5 \\
\hline Camión cisterna & $21(14,8)$ & 9,4 a 21,7 \\
\hline Aguatero & $19(13,4)$ & 8,2 a 20,1 \\
\hline Caño fuera del domicilio por red pública & $4(2,8)$ & 0,7 a 7,0 \\
\hline Pileta & $3(2,1)$ & 0,4 a 6,1 \\
\hline \multicolumn{3}{|l|}{ Almacena el agua por más de 3 días en: } \\
\hline Cilindros o bidones & $46(32,4)$ & 24,8 a 40,7 \\
\hline Tanques bajos & $30(21,1)$ & 14,7 a 28,7 \\
\hline Tanques elevados o cisternas & $7(4,9)$ & 2,0 a 9,8 \\
\hline No almacena & $59(41,5)$ & 33,3 a 50,1 \\
\hline
\end{tabular}


del vector, el 29,6\% (42; IC95\% 22,2 a $37,8)$ de los alumnos reconocieron las larvas del vector, y tampoco se encontraron diferencias en el género y en el nivel educativo.

Respecto a las actitudes, si algún miembro de la familia presentara fiebre, $46,4 \%$ (66) de los entrevistados acudiría a un establecimiento del primer nivel de atención del Ministerio de Salud (MINSA) o EsSalud y 42,9\% (61) a un policlínico u hospital del MINSA o de EsSalud. Asimismo, 83,1\% (118) de los estudiantes aceptaría realizarse un examen serológico para descartar dengue en el caso de presentar fiebre.

El 57,7\% (82; IC95\% 49,1 a 65,9) indicaron que sus jefes de familia permitirían que el personal de salud ingrese a sus viviendas para la búsqueda del vector o realice alguna intervención. El $75,4 \%$ (75; IC95\% 67,4 a 82,2$)$ aceptaría participar en la vigilancia del vector y existieron diferencias significativas entre los niños y las niñas $(p=0,049238)$. El 37,3\% (53) de los alumnos percibía que el responsable de prevenir o controlar el dengue en la comunidad sería el MINSA, el 18,3\% (26) los puestos o centros de salud, el 15,5\% (22) la Municipalidad y el 9,9\% (14) EsSalud. A 97,9\% (139) de los estudiantes les gustaría recibir información sobre el dengue, de los cuales $64,7 \%$ (90) prefería recibirla en su colegio y $25,8 \%$ (36) en un puesto o centro de salud.

Respecto al antecedente de inspección domiciliaria por parte del personal de vigilancia y control vectorial, solo $29,5 \%$ (42; IC95\% 22,2 a 37,8) de los alumnos había recibido la visita del personal de salud en los últimos 8 meses en sus viviendas para la colocación de Abate $^{\circledR}$ (larvicida) y/o brindarles información. De aquellos que recibieron la visita, 45,2\% (19/42) refirió que los depósitos de agua en su vivienda contenían el larvicida al momento de la encuesta.

En cuanto a las prácticas de prevención y control de dengue, los resultados sobre las medidas para disminuir el ries- go de la reproducción y la picadura del vector, así como, el abastecimiento y el almacenamiento de agua se muestran en la tabla 1. El 35,9\% (51; IC95\% 28,0 a 44,4$)$ de los alumnos refirieron que por lo menos un familiar había viajado fuera del departamento de Lima en los últimos 6 meses y los departamentos más visitados fueron Junín y Cusco $(13,7 \%)$, seguidos por Ancash (11,8\%) y Huánuco $(9,8 \%)$.

Por otro lado, el 47,9\% (68; IC95\% $39,4$ a 56,4$)$ de los alumnos mencionaron haber recibido información previa sobre dengue por algún medio durante el año. De estos, $21,8 \%$ (31) recibió la información en el colegio, $19,7 \%$ (28) por la televisión, 9,1\% (13) en los puestos o centros de salud, 3,5\% (5) a través de la familia y $1,4 \%$ (2) por medio de los periódicos y la radio.

En el análisis bivariado, el no haber recibido información previa se asoció a no tener conocimiento básico de dengue $(\mathrm{OR}=3,7$; IC95\% 1,7 a 8,3$)(\mathrm{p}=$ $0,0004676)$.

Finalmente, los dibujos realizados por los alumnos brigadieres sobre la forma de transmisión de dengue, el ciclo biológico del vector y las condiciones para la transmisión se observan en las figuras 1,2 y 3 .

\section{DISCUSIÓN}

Este es el primer estudio de percepción que se realiza en escolares en el Perú en una zona de infestación aédica (Escenario II). A pesar de ello, la tercera parte de los alumnos de educación primaria tuvieron conocimientos básicos de dengue (fiebre más un signo o síntoma y la identificación correcta de las formas de transmisión). Es probable que la difusión de mensajes y las noticias en los medios de comunicación durante el brote de dengue en el distrito de Villa María del Triunfo ocurrida el 2013, habría contribuido a estos resultados.

La frecuencia de los conocimientos sobre la identificación de los signos y síntomas correctos del dengue son similares a los escolares de 12 a 16 años de educación secundaria de Bangkok, Tailandia ${ }^{(23)}$; sin embargo, es muy baja comparada con otros estudios ${ }^{(15-20)}$. Esta diferencia puede explicarse porque el distrito de Chorrillos tenía apenas siete meses de haberse infestado al momento del estudio; además, no es una zona endémica para dengue y porque la metodología para medir el conocimiento fue diferente en los estudios previos ${ }^{(15-20)}$. La identificación de las formas de transmisión en los escolares fue más alta que en los pre-escolares y escolares de Neiva y Huila en Colombia ${ }^{(15)}$, pero más bajas que en los

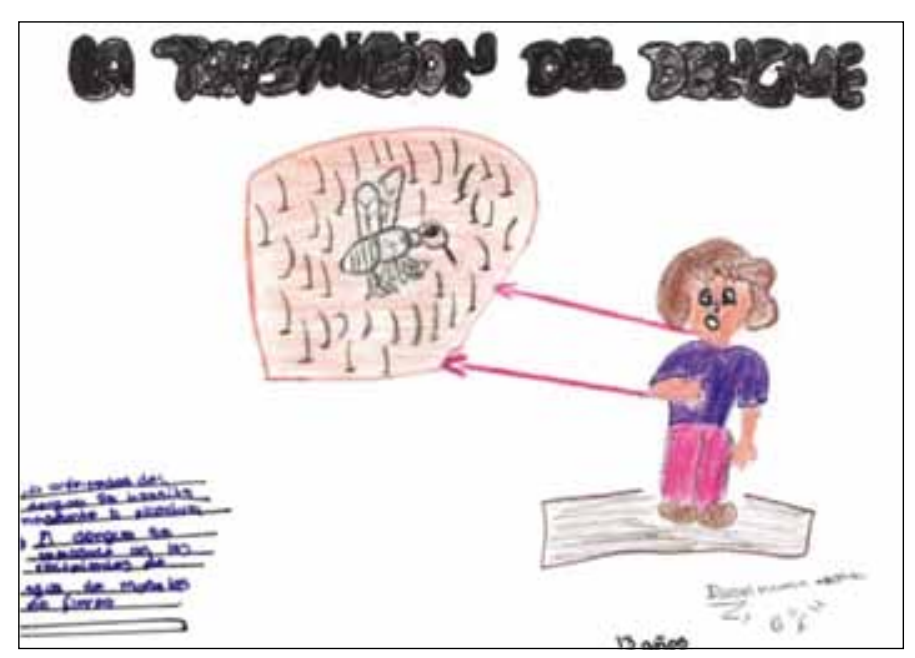

Figura 1. Forma de transmisión del dengue, dibujo de una niña de 13 años de sexto grado. 


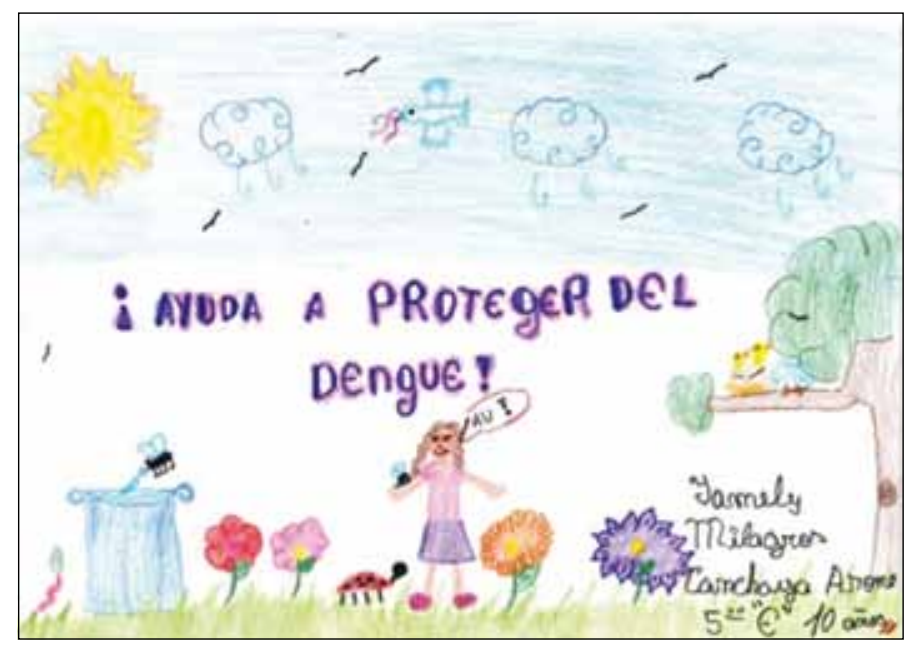

Figura 2. Almacenamiento de agua como factor de riesgo para la infestación por el vector del dengue, dibujo de una niña de 10 años de quinto grado.

estudiantes del estado de Maranhão, Brasil (17), Chiapas, México ${ }^{(18)}$ y Tailandia ${ }^{(20,23)}$; sin embargo, en los dibujos realizados por los brigadieres, está muy claro que el dengue es transmitido por la picadura de los mosquitos. A pesar de ello, es importante enfatizar las formas de transmisión en todos los espacios, como los colegios, en las visitas domiciliarias y en los mensajes que se difunden en los medios de comunicación social.

Por otro lado, los síntomas más reconocidos fueron la fiebre y cefalea, como en una zona endémica de Arabia Saudita, con diferencias muy marcadas en la proporción del reconocimiento del rash, hemorragia, shock y muerte ${ }^{(19)}$, por ser un escenario diferente; se explicaría por la intensidad de transmisión del área de estudio con una tendencia a la severidad y por tratarse de escolares con mayor nivel educativo ${ }^{(19)}$. No obstante, las dos terceras partes consideraron al dengue como una enfermedad grave. En este reporte, una elevada proporción de escolares reconocieron los síntomas en forma aislada; sin embargo, estos síntomas o signos pueden presentarse en cualquier otra enfermedad.

Por otro lado, la mitad de los encuestados reconocieron la forma adulta de Aedes aegypti, pero solo un tercio distin- guió las larvas y pupas. Esto podría estar relacionado a una mayor difusión de imágenes de la forma adulta del vector más no de las larvas ${ }^{(9)}$. En los dibujos realizados por los brigadieres, no tienen claro los estadios en el ciclo biológico del vector, que es entendido como ciclo del dengue, y confunden las larvas del vector con las larvas de las moscas. Por ello, es necesario que en los mensajes se pueda diferenciar las larvas de Aedes de otros dípteros y enfatizar el ciclo completo. En otras regiones endémicas, como Bucaramanga, Colombia, los es- colares reconocen mejor las larvas que los adultos ${ }^{(24)}$. La identificación correcta del vector y el comportamiento por los escolares es clave en los programas de vigilancia entomológica comunal.

La mayoría de los escolares reconocieron el ecótopo del vector, que guarda relación con los tipos de criaderos para Aedes aegyti en la ciudad de Lima. Esto también es expresado en la técnica proyectiva por los alumnos sobre las condiciones ambientales para la infestación aédica. El reconocimiento del uso del larvicida como medida de prevención de la infestación vectorial fueron bajas, así como, el cambio de arena por agua de los floreros y el recambio de agua frecuente. Estas medidas deben enfatizarse en los mensajes a la población en riesgo, sobre todo, en áreas en escenario sin transmisión autóctona como Chorrillos. Asimismo, aún fue más baja la proporción de conocimientos de las medidas para evitar la picadura del vector adulto. En otras regiones endémicas los escolares conocen mejor el uso de mosquiteros, repelentes comerciales o inclusive repelentes naturales ${ }^{(25)}$.

Respecto a las actitudes, menos de la mitad de los escolares en el caso de tener fiebre acudirían a un establecimiento del primer nivel de atención del Ministerio de Salud (MINSA) o

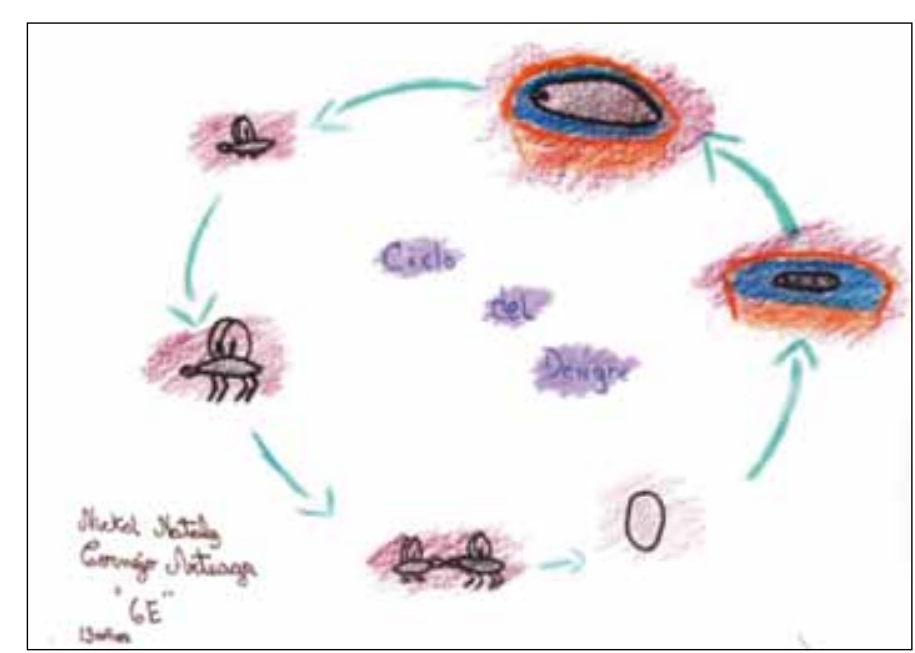

Figura 3. Ciclo de vida del vector del dengue, dibujo de una niña de 13 años de sexto grado. 
EsSalud. Este hallazgo refleja la necesidad de mejorar los mensajes para que acudan oportunamente a los servicios de salud de atención primaria, lo que permitiría la detección oportuna de los casos importados o brotes de dengue. Asimismo, la proporción de escolares que manifestaron que sus padres dejarían ingresar al personal de salud para realizar las intervenciones fue muy baja. Este es uno de los problemas críticos en el control focal y espacial ${ }^{(6-7)}$ y plantea la necesidad de realizar otros estudios cualitativos como el focus group y entrevistas de profundidad para identificar las causas del problema.

Más de dos tercios de los estudiantes con un predominio en los niños estaban dispuestos a participar en un eventual programa de vigilancia entomológica comunal. Esta estrategia debe implementarse dado el limitado número de recursos humanos para esta actividad, el recambio constante de los inspectores, el número grande de viviendas bajo vigilancia y el incremento sostenido de nuevas localidades y distritos infestados. Existen experiencias locales en algunas regiones del Perú de participación de los estudiantes durante las campañas de recojo de criaderos y en actividades de difusión de las medidas de prevención y control. El Ministerio de Educación ha regulado actividades de prevención de la infestación aédica dentro de las instituciones educativas del país en los tres niveles ${ }^{(26)}$; sin embargo, estas actividades también deben realizarse en la vivienda y la comunidad y requiere una intervención educativa con una activa participación de los estudiantes en actividades de vigilancia, prevención y control $(18,20,27,28)$. La prevención y el control del dengue requieren una efectiva participación multisectorial, que tampoco es percibida por los estudiantes. En un estudio antropológico en población adulta en México se han identificado 3 barreras que obstaculizan las actividades de prevención; la información confusa e insuficiente sobre dengue, la atribución de la responsabilidad de prevenir a otras personas u organismos públicos y la ex- cesiva confianza en la fumigación como medida preventiva ${ }^{(29)}$.

En este estudio se evidencia que existen potenciales determinantes para la infestación aédica en las viviendas de los estudiantes y para la instalación de brotes de dengue o de la fiebre de chikungunya, debido a que más del $30 \%$ de los escolares encuestados no tenían agua dentro del domicilio, más de $58 \%$ almacenaba agua por más de 3 días, más del 10\% de los padres de los escolares trabajaban en otros distritos con infestación aédica y existieron evidencias de viajes fuera de la ciudad de Lima en los últimos 6 meses a departamentos con transmisión de dengue. Los patrones de movilización humana tienen un impacto crítico en la propagación de la transmisión de dengue ${ }^{(30)}$.

Un hallazgo interesante es que los escolares recibieron más información en los colegios y a través de la televisión; esto es muy importante para difundir los mensajes sobre las medidas de prevención y control del dengue en esta población. Y el antecedente de no recibir información se asoció al desconocimiento de la enfermedad.

Una limitación del estudio es que no se ha considerado una visita domiciliaria a la vivienda de los escolares; sin embargo, es un estudio que combina un estudio epidemiológico con un enfoque cualitativo (técnicas proyectivas) para explorar mejor la percepción de los escolares sobre el dengue. Se requieren más estudios en otras regiones endémicas para explorar la percepción de los escolares a fin de incorporarlos en las actividades de vigilancia, prevención y control del dengue.

Los conocimientos de los estudiantes sobre los signos y síntomas y la forma de transmisión del dengue, las características del vector y las medidas de prevención del dengue son bajos y el no recibir información se asocia al desconocimiento del dengue. Asimismo, existen prácticas de riesgo que favorecen la potencial presentación de brotes de dengue.

\section{AGRADECIMIENTOS}

Al Dr. Luis Suárez Ognio, coordinador del curso de Epidemiología de la Universidad Peruana de Ciencias Aplicadas, por las facilidades brindadas para realizar el presente estudio. Al profesor Oscar Zambrano Raymi, director de la Institución Educativa Juan Pablo II 7075, por brindar las facilidades para desarrollar el presente estudio.

\section{REFERENCIAS BIBLIOGRÁFICAS}

1. Murray NEA, Quam MB, Wilder-Smith A. Epidemiology of dengue: past, present and future prospects. Clin Epidemiol. 2013:5:299-309. doi: 10.2147/ CLEP.S34440.

2. World Health Organization (WHO). Global strategy for dengue prevention and control 2012-2020. Geneva: WHO; 2012.

3. Philips I, Need J, Escamilla J, Colan E, Sánchez S, Rodriguez M, et al. First documented outbreak of dengue in the Peruvian Amazon region. Bull Pan Am Health Organ. 1992;26(3):201-7.

4. Andrade CS, Cáceres AG, Vaquerizo A, IbañezBernal S, Cachay LS. Reappearance of Aedes aegypti (Diptera: Culicidae) in Lima, Peru. Mem Inst Oswaldo Cruz. 2001;96(5):657-8.

5. Pachas P, Sarria M, Garcia MP, Suárez-Ognio L, Avila J, Munayco C, et al. Magnitude of the first outbreak of dengue fever in district of Comas, LimaPeru. Abstract Book American Society of Tropical Medicine and Hygiene 55th Annual Meeting. 12-16 november 2006. Atlanta, USA. p. 792

6. Perú, Ministerio de Salud. Brote de dengue en el distrito Puente Piedra, Lima, 2012. Bol Epidemiol (Lima). 2012; 21(12):208.

7. Dirección de Salud II Lima Sur. Primer brote de Dengue en Lima Sur. Distrito de Villa Maria del Triunfo, 2013. Lima: DISA Lima Sur; 2013.

8. Perú, Ministerio de Salud. Conocimientos, actitudes y prácticas de la población frente al dengue: Lima y Macro Región Norte del Perú. Lima: Ministerio de Salud, Dirección General de Promoción de la Salud, Proyecto Vigia, 2004.

9. De la Cruz, AM, Figueroa D, Chacón L, Gómez M, Diaz M, Finlay CM. Conocimientos, opiniones y prácticas sobre Aedes aegypti. Rev Cubana Med Trop. 1999;51(2):135-7.

10. Claro LBL, Barbosa HC, Garcia ML. Prevenção e controle do dengue: uma revisão de estudos sobre conhecimentos, crenças e práticas da população. Cad Saúde Pública. 2004;20(6):1447-57.

11. Chiaravalloti Neto F. Conhecimentos da população sobre dengue, seus vetores e medidas de controle em São José do Rio Preto, São Paulo. Cad Saúde Pública. 1997;13(3):447-53.

12. Schweigmann N, Rizzotti A, Castiglia G, Gribaudo $F$, Marcos E, Burroni $N$ y col. Información, conocimiento y percepción sobre el riesgo de contraer el dengue en Argentina: dos experiencias de intervención para generar estrategias locales de control. Cad Saúde Pública. 2009;25(Suppl.1):S137-48.

13. Ventosilla P, Torres E, Harman L, Saavedra K, Mormontoy W, Merello J y col. Conocimientos, actitudes y prácticas en el control de malaria y 
dengue en las comunidades de Salitral y Querecotillo, departamento de Piura. Mosaico Cient. 2005;2(2):65-9.

14. Jamanca SR, Touzett VA, Campos AL, Jave $\mathrm{CH}$, Carrión MM, Sánchez CS. Estudio CAP de dengue en los distritos de cercado de Lima, La Victoria y San Luis. Lima, Perú. Junio 2004. Rev Peru Med Exp Salud Publica. 2005;22(1):26-31.

15. Fajardo P, Monje CA, Lozano G, Realpe O, Hernández LE. Nociones populares sobre "dengue" y "rompehuesos", dos modelos de la enfermedad en Colombia. Rev Panam Salud Pública. 2001;10(3):161-8.

16. Combina VMV. Determinación del nivel de conocimientos sobre dengue en alumnos de escuelas municipales de la ciudad de Córdova, Argentina. Perspectivas para la prevención. Rev Salud Pública. 2008;2(1):37-51.

17. Bezerra JM, Silva JS, Ibiapina SS, Tadei WP, Pinheiro VC. Evaluation of students' knowledge as a contribution to dengue control programs. Cien Saude Colet. 2011;16(11):4367-73.

18. Torres JL, Ordóñez JG, Vázquez-Martinez MG. Conocimientos, actitudes y prácticas sobre el dengue en las escuelas primarias de Tapachula, Chiapas, México. Rev Panam Salud Publica. 2014;35(3):214-8.

19. Ibrahim NK, Al-Bar A, Kordey M, Al-Fakeeh A. Knowledge, attitudes, and practices relating to Dengue fever among females in Jeddah high schools. J Infect Public Health. 2009;2(1):30-40.

20. Suwanbamrung $S$. Children's basic knowledge and activities for dengue problem solution: an islamic religious school, Southern Thailand. Asian Pac J Trop Dis. 2012;2(6):456-64.

21. Perú, Instituto Nacional de Estadistica e Informática (INEI). Perú: Estimaciones y proyecciones de población por sexo, según departamento, provincia y distrito, 2000-2015. Bol Especial № 18, 2009.

22. Perú, Instituto Nacional de Estadistica e Informática (INEI). Perú: Mapa del déficit de agua y saneamiento básico a nivel distrital, 2007. Lima: INEI; 2010.

23. Chanyasanha C, Han MM, Teetipsatit S. Dengue hemorrhagic fever knowledge, perception, and preventive behavior among secondary school students in Bangkok. J Med Assoc Thai. 2013;96 Suppl 5:S14-24.

24. Vesga-Gómez C, Cáceres-Manrique FM. Eficacia de la educación lúdica en la prevención del Dengue en escolares. Rev salud pública. 2010;12(4):558-69.

25. Suwanbamrung C, Promsupa S, Doungsin T, Tongjan S. Risk factors related to dengue infections in primary school students: Exploring students' basic knowledge of dengue and examining the larval indices in southern Thailand. J Infect Public Health. 2013;6(5):347-57. doi: 10.1016/j.jiph.2013.04.006.

26. Aprueban "Normas para orientar la organización y desarrollo de las actividades de educación preventiva, mitigación y respuesta ante el dengue en las instituciones educativas públicas y privadas del país". Resolución Ministerial Nº 0493-2012-ED. El Peruano, 2012;XXIX(12286):480309-10. (8 de diciembre de 2012).

27. Jayawardene WP, Lohrmann DK, YoussefAgha $\mathrm{AH}$, Nilwala DC. Prevention of dengue fever: An exploratory school-community intervention involving students empowered as change agents. J Sch Health. 2011;81(9):566-73. doi: 10.1111/j.17461561.2011.00628.x.

28. Khun S, Manderson L. Community and schoolbased health education for dengue control in rural Cambodia: a process evaluation. PLoS Negl Trop Dis. 2007;1(3):e143.

29. Torres López TM, Guerrero Cordero JL, Salazar Estrada JG. Dimensiones culturales del dengue que favorecen o dificultan su prevención en México. Rev Panam Salud Publica. 2012;31(3):197-203.

30. Stoddard ST, Forshey BM, Morrison AC, PazSoldan VA, Vazquez-Prokopec GM, Astete $\mathrm{H}$, et al. House-to-house human movement drives dengue virus transmission. Proc Natl Acad Sci USA. 2013;110(3):994-9. doi: 10.1073/ pnas.1213349110.

Articulo recibido el 29 de diciembre de 2015 y aceptado para publicación el 21 de febrero de 2016.

Este estudio forma parte de los trabajos extramurales de los cursos de Epidemiología y Médico Paciente 6. Escuela de Medicina, Universidad Peruana de Ciencias Aplicadas, Lima, Perú.

Fuente de financiamiento: Autofinanciado.

Conflictos de interés: R. Cabrera fue coordinador del Grupo Temático de Enfermedades Trasmitidas por Vectores en la Dirección General de Epidemiología, Ministerio de Salud. El resto de los autores declaran no tener conflictos de interés en la publicación de este artículo.

Correspondencia:

Rufino Cabrera.

Escuela de Medicina, Universidad Peruana de Ciencias Aplicadas.

Av. Alameda San Marcos, Cuadra 2, Chorrillos, Lima, Perú.

Correo electrónico: pcmercab@upc.edu.pe; rcabrerach@hotmail.com 